\title{
Lysosome Depletion-Triggered Autophagy Impairment in Progressive Kidney Injury
}

\author{
Xiao-cui Chen ${ }^{a}$ Zhi-hang Li ${ }^{\mathrm{a}}$ Chen Yang $^{\mathrm{a}}$ Ji-xin Tang ${ }^{\mathrm{a}}$ Hui-yao Lan ${ }^{\mathrm{b}}$ \\ Hua-feng Liu ${ }^{a}$
}

${ }^{a}$ Key Laboratory of Prevention and Management of Chronic Kidney Disease of Zhanjiang City, Institute of

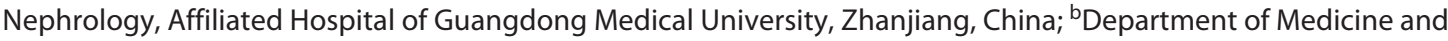
Therapeutics and Li Ka Shing Institute of Health Sciences, The Chinese University of Hong Kong, Hong Kong SAR, China

\section{Keywords}

Autophagy · Lysosome · Kidney disease - Tubular epithelial cell · Podocyte

\section{Abstract}

Background: Macroautophagy (autophagy) is a cellular recycling process involving the destruction of damaged organelles and proteins in intracellular lysosomes for efficient nutrient reuse. Summary: Impairment of the autophagy-lysosome pathway is tightly associated with multiple kidney diseases, such as diabetic nephropathy, proteinuric kidney disease, acute kidney injury, crystalline nephropathy, and drug-and heavy metal-induced renal injury. The impairment in the process of autophagic clearance may induce injury in renal intrinsic cells by activating the inflammasome, inducing cell cycle arrest, and cell death. The lysosome depletion may be a key mechanism triggering this process. In this review, we discuss this pathway and summarize the protective mechanisms for restoration of lysosome function and autophagic flux via the endosomal sorting complex required for transport (ESCRT) machinery, lysophagy, and transcription factor EB-mediated lysosome biogenesis. Key Message:

karger@karger.com www.karger.com/kdd

Karger $\stackrel{\text { ' }}{=}$
(C) 2021 The Author(s)

Published by S. Karger AG, Basel

This is an Open Access article licensed under the Creative Common Attribution-NonCommercial-4.0 International License (CC BY-NC) (http://www.karger.com/Services/OpenAccessLicense), applicable to the online version of the article only. Usage and distribution for commercial purposes requires written permission.
Further exploring mechanisms of ESCRT, lysophagy, and lysosome biogenesis may provide novel therapy strategies for the management of kidney diseases.

(C) 2021 The Author(s)

Published by S. Karger AG, Basel

\section{Introduction}

Macroautophagy is an evolutionarily conserved lysosome-dependent catabolic process that sequesters the cytoplasmic materials, such as protein aggregates and damaged organelles, into a double-membrane structure termed autophagosome for lysosome degradation. This process is crucial to maintain the cellular homeostasis in eukaryotic cells. In addition to macroautophagy, chaperone-mediated autophagy and microautophagy are 2 other types of autophagy that vary based on the form of substrate delivery [1]. Macroautophagy, generally referred to as autophagy, is also classified as "basal autophagy" or "induced autophagy." In contrast to spontaneous basal

Xiao-cui Chen and Zhi-hang Li contributed equally to this work.
Correspondence to:

Hui-yao Lan, hylan@cuhk.edu.hk

Hua-feng Liu, hf-liu@263.net 
autophagy, induced autophagy can be triggered by cellular stresses including nutrient starvation, oxidative stress, hypoxia and endoplasmic reticulum (ER) stress, or other harmful stimuli [2].

Autophagy consists of several steps, including initiation, phagophore nucleation, autophagosome formation, lysosome fusion and degradation $[3,4]$. Lysosomes are single-membrane organelles, containing over 50 acid hydrolases. Attributed to its acid hydrolases and strong acidic luminal $\mathrm{pH}$, lysosomes are supposed to the shared degradative compartments of endocytic and autophagic pathways [5]. Lysosome fusion with autophagosomes leads to the breakdown of phagocytosed cellular components by exposing them to acidic hydrolases. The degraded products generated by lysosomal hydrolases are then reused for synthesis of new cellular components and energy, which are essential for cell survival, differentiation, development, homeostasis, and energy production [6].

Increasing evidence has shown that lysosome disruption (such as induced by nephrotoxin) may not only impair the autophagic clearance but also triggers cell death, which may contribute to the progress of various kidney diseases. In order for kidney cells to survive, damaged lysosomes must be cleared via autophagy-dependent pathway (lysophagy), and the impaired degradation capacity must be restored through lysosome biogenesis.

In this review, we discuss the autophagy-lysosome pathway and summarize the protective mechanisms for restoration of lysosome function and autophagic flux via lysophagy and transcription factor EB (TFEB)-mediated lysosome biogenesis. Further exploration of these mechanisms may provide novel therapy strategies for the management of kidney diseases through targeting autophagylysosome pathway.

\section{The Autophagy Process}

\section{Initiation of Autophagosome Formation}

The formation of autophagosome is a multistage process triggered by activation of the Unc-51-like kinase 1 (ULK1) complex (ULK1/2, ATG13, FIP 200, and ATG101) and the class III phosphatidylinositol 3-kinase (PI3K) complex that consists of VPS34, Beclin1, UVRAG, Bif1, and P150, which promotes the nucleation of the phagophore membrane. Coat protein complex II (COPII)-coated vesicles, bud from specialized regions of the ER called ER exit sites, are known to help for autophagosomes formed and are necessary for ER-Golgi traffic and autophagy. Under nutrient-rich conditions, COPII vesicles mediate ER-Golgi traffic. However, under starvation or stress conditions, COPII-coated vesicles are contributed to autophagosomes formation [7]. Autophagosome biogenesis is initiated from the generation of phagophore, which then expands into an autophagosome [8]. Subsequently, the conjugation of ATG5-ATG12 complex and ATG16 elongates the autophagosome membrane, and concomitantly, a cytosolic form of LC3-I conjugates with phosphatidylethanolamine to generate lipidatedLC3-IIthat attachestotheexpandingautophagosome membrane [3]. Finally, completing autophagosome formation after autophagy cytoplasmic cargo is engulfed.

In bulk autophagy, the formation of autophagosome is generally controlled by mammalian target of rapamycin complex 1 (mTORC1) and AMP-activated protein kinase (AMPK). In response to growth factors and nutrients, activation of mTORC1 promotes the cellular anabolic processes but suppresses the catabolic processes by blocking autophagy via phosphorylation of ULK1 and its cooperator ATG13 $[9,10]$. In contrast, AMPK, an energy-sensitive enzyme, can be activated by high AMP/ATP ratios and then suppresses mTORC1 by phosphorylated tuberous sclerosis complex 2 . In addition, AMPK can directly phosphorylate ULK1 at the other sites to activate the autophagy pathway. Indeed, inhibition of mTORC1 by tizoxanide [11] or rapamycin [12] triggers autophagy, whereas suppression of AMPK abolishes ULK1-mediated autophagy initiation [13]. Moreover, the regulatory mTOR-dependent autophagy is also influenced by activation of PI3K/AKT and protein kinase B signaling.

Autophagy has been shown to be initiated by specifically endogenous danger signals. This process may be independent of AMPK/mTORC1. Specific molecules sense the damaged organelles and recruit the ubiquitin ligase to modulate the ubiquitination and then establish a platform to activate the ULK1-mediated core autophagy machinery. For example, mitophagy involves in selective removal of the damaged mitochondria via an autophagosome-lysosome-dependent pathway. When mitochondrial damage occurs, PTEN-induced kinase 1 (PINK) recruits and activates the E3 ubiquitin ligase Parkin, which then tags mitochondrial surface proteins by ubiquitination and recruits autophagic adapter proteins, such as p62 [14]. Subsequently, the core autophagy machinery is activated to engulf the damaged mitochondria into the autophagosome for lysosome-mediated degradation. A similar process also occurs for selective degradation of the peroxisomes, ER and nucleus via an autophagosome-lysosome-dependent pathway [15]. 
Transport of Autophagosomes and Autophagosome-

Lysosome Fusion

Lysosomes are mainly concentrated in the perinuclear region. Once autophagosome formation is completed, it must be delivered to the perinuclear region to fuse with lysosomes for digestion. This is a key step in maintaining autophagy flux [16, 17]. Autophagosomes are delivered along with the cytoskeleton, including microtubules and actin filaments. The minus-end-directed dynein-dynactin motor complex mediates the movement of autophagosomes to the perinuclear region, whereas the plus-enddirected motor assists to move the autophagosomes to the cell peripheral region [16]. Interestingly, antibodies against LC3 disrupt the efficient movement of autophagosomes, indicating the involvement of LC3 in this process [18]. The rate of autophagosomal fusion is also determined by the localization of lysosomes. Increased perinuclear localization of lysosomes promotes autophagosomal fusion and vice versa [19]. The movement of autophagosomes into the perinuclear region reaches the vicinity of lysosomes and must then be tethered to the lysosomes. Next, SNARE proteins carry out autophagosome-lysosome fusion. This process has been described in detail in recent reviews $[16,20]$.

\section{Lysosome-Dependent Degradation}

Lysosome plays a key role in the degradation of damaged organelles, aggregated proteins and pathogens. And it acts as a key cellular catabolic center for fundamental metabolic and signaling functions [21, 22]. Lysosomes are created from Golgi apparatus, whereas the hydrolases are manufactured in the ER. After decorating with mannose6-phosphate, these hydrolytic enzymes are delivered to the Golgi apparatus by transport vesicles and then packaged into lysosomes. In general, lysosomal membrane is resistant to digestion since glycosylated membrane proteins are specifically expressed in the lysosomes, such as lysosomal-associated membrane protein (LAMP)-1 and LAMP-2, and function to protect them from degradation by acidic hydrolases [23]. Furthermore, other membrane proteins such as transporter protein, ion channel protein, and SNARE protein are involved in mediating different aspects of lysosome functions, especially the vATPase complex which mediates the acidification of lysosome and regulates the fission and fusion of lysosomes [24].

When autophagosomes fuse with lysosomes, autolysosomes are formed. The inner autophagosomal membrane and engulfed cargo are then digested by lysosomal hydrolytic enzymes [25]. The catabolites generated from autophagosome degradation may be exported from lyso- somes to the cytoplasm for the reuse to form new macromolecules. However, the relevant transporters and mechanisms for amino acids and lipids are largely unknown [26]. After degradation of autophagic substrates, the amino acid levels in the cytoplasm increase and mTORC1 is activated. The reactivation of $\mathrm{mTORC} 1$ not only negatively regulates autophagy to avoid overactivation but also helps lysosomes regenerate from autolysosomes. Impairment of this process, termed autophagic lysosome reformation, makes the cells highly susceptible to starvation-induced cell death [27].

\section{Impairment of Autophagosome Clearance and Depletion of Lysosomes}

\section{Impairment of Autophagosome Clearance by}

Lysosome Depletion

Autophagy plays crucial roles in preserving cellular homeostasis. Interruption of autophagy is implicated in various human diseases. However, compared with the initiation steps, the later steps of autophagosome-lysosome fusion and degradation have not been extensively characterized. Ma et al. [28] reported that accumulation of autophagosomes is associated with elevated reactive oxygen species (ROS) and mitochondrial permeabilization, resulting in cardiomyocyte death. In addition, Sarkar et al. [29] showed that after traumatic brain injury, autophagosome clearance is impaired and is correlated with neuronal cell death. Consistent with this finding, Cui et al. [30] demonstrated that accumulation of autophagosomes increases the caspase-dependent and independent apoptosis, as well as necrosis in neurons, in a model of neonatal hypoxic-ischemic encephalopathy. Interestingly, initiation of autophagy and autophagosome formation is not inhibited but instead enhanced.

Typically, impairment of autophagosome clearance is related to lysosome depletion. It can result from the existing lysosome dysfunction or the impairment of lysosome biogenesis (as described in detail below). Lysosome dysfunction may be caused by the deficiencies of lysosomal enzymes, elevated lysosomal $\mathrm{pH}$, the changes in lysosome intracellular localization, and increased lysosomal membrane permeability. For example, Wang et al. [31] found that lysosome dysfunction induced by silica nanoparticles inhibits autophagosome degradation, resulting in the disruption of autophagy flux Mounting evidence suggests the involvement of lysosome depletion in various human diseases including lysosomal storage diseases, neurodegenerative diseases, autoimmune diseases, and diabetes [23, 32, 33] 
Lysosomal Membrane Permeabilization as the Main Manifestation of Lysosome Depletion

Lysosomal membrane integrity is essential for lysosome function. Lysosomal membrane permeabilization (LMP) leads to the leakage of lysosomal contents then results in lysosome dysfunction, thus impairing autophagosome clearance. In addition, the leakage of lysosomal constituents is thought to be sufficient to trigger cell death in a caspase-dependent or independent manner [34,35]. However, partial and selective disruption of lysosomes results in cell apoptosis [36]. Moreover, the extracellular signal-regulated kinase pathway is necessary for promoting lysosome-mediated cell death [37]. Several reports have indicated that the membrane permeabilization is not observed in all lysosomes at the same time in response to some lethal stimuli. Although the mechanisms related to this heterogenetic observation remains unclear, it seems that large lysosomes are particularly sensitive to the effects of LMP-inducing agents $[23,38]$.

LMP can be triggered by indigestible substrates and the intralysosomal ROS. It can also be regulated by transcription factor p53 in a transcription-dependent or independent manner. Some reports suggested that the phosphorylated p53 at Ser15 upon treatment with tumor necrosis factor (TNF)- $\alpha$, radiation, or DNA-damaging drugs can result in it translocated to the lysosomal membrane and consequently induces LMP $[39,40]$. Additionally, it has been found that the transcription factor signal transducer and activator of transcription 3 is involved in inducing LMP, which can upregulate the lysosomal proteases cathepsin B and $\mathrm{L}$ and downregulate the lysosome endogenous inhibitor Spi2A [41]. Various proteases, such as caspases, cathepsins, and calpains, are also involved in LMP. Guicciardi et al. [42] showed that caspase- 8 can promote the release of cathepsins from lysosomes, and this process is enhanced after induction of LMP. Of note, lysosomal cathepsin B could constitutively amplify the feedback loop to stimulate the release of their own from the lysosome to the cytoplasm and TNF- $\alpha$ induces LMP via a cathepsin B-dependent manner [43]. Consistently, overexpression of the endogenous cathep$\sin$ B inhibitor protein Spi2A can reduce LMP induced by TNF- $\alpha$ [44]. Thus, once lysosome is damaged, it could represent a potential hazard to the cell.

Autophagy plays a crucial role in several kidney diseases. The regulation and function of autophagy in kidney diseases is likely dependent on cell types and disease conditions because of high levels of basal autophagy in kidney cells $[45,46]$. In this section, we focus on studies in kidney diseases associated with disruption of autoph- agy caused by lysosome depletion and impairment of autophagic clearance.

\section{Diabetic Nephropathy}

Diabetic nephropathy (DN) is a serious kidney-related complication in patients with type 1 and type 2 diabetes [32, 47-50]. Hyperglycemia mediators such as advanced glycation end products (AGEs), protein kinase C, and glucose play a crucial role in the pathogenesis of DN. Autophagy deficiency or insufficiency in renal intrinsic cells including podocytes and tubular epithelial cells (TECs) is also involved in the pathogenesis of DN [51]. Tagawa et al. [52] found that large accumulation of $\mathrm{p} 62$ protein is observed in the glomeruli in kidney biopsy sample of patients with DN. Moreover, they also found that in DN rat model with severe proteinuria, a reduction in podocinpositive areas and alteration in foot processes are observed in podocytes, accompanied by an increase in p62 and a decrease in LC3-II [52]. Similarly, another group showed that the injurious podocytes in $\mathrm{DN}$ are closely associated with the decreased autophagy flux as demonstrated by the accumulation of $\mathrm{p} 62$ and the interaction between p62 and LC3 [53]. Additionally, impaired autophagy in podocytes can also result in the podocyte loss, which ultimately promotes proteinuria and DN development $[54,55]$. In our previous study, we showed that AGEs overload disrupts the autophagic pathway in podocytes due to LMP [30]. Furthermore, Atsushi and colleagues found that autophagy promotes AGEs degradation in the diabetic kidney by regulating lysosome biogenesis. Activation ofautophagycan reduceinflammasome activation and improve kidney fibrosis [56]. Another study suggested that autophagy impairment and lysosome dysfunction occur in the cultured podocytes treated with sera from patients and rats with diabetes and massive proteinuria, leading to apoptosis [51].

Autophagy activation is a mechanism that protects renal TECs from injury in DN. However, the impairment of autophagy-lysosome pathway also causes renal tubular injury in DN [32]. Several studies have shown that hyperglycemia inhibits the activation of autophagy, whereas increased expression levels of p62 is also evidenced in proximal tubular cells in animal models of both type 1 and type 2 diabetes [51,57]. In our study, we found that AGEs block the autophagic flux and significantly decrease the lysosomal activity and degradative potential in TECs [32]. Furthermore, our current study found that Smad3 activation mediates AGEs-induced autophagic flux blockage since Smad3 can directly bind to the $3^{\prime}$-UTR of TFEB and inhibit its transcription, thus leading to the de- 
ficiency of lysosome biogenesis [58]. Taken together, these findings support that the injury of renal intrinsic cells in DN is associated with impaired autophagic flux and lysosome homeostasis.

\section{Proteinuric Kidney Disease}

Proteinuric kidney diseases are a set of renal disorders characterized by the presence of massive proteins in the urine. Proteinuria is not only a marker of kidney diseases but also an independent risk factor for the loss of renal function. Most proteinuric kidney diseases are attributed to change in the structure and function of podocytes, which are the key components of glomerular filtration barrier.

Several studies have shown that proteinuria caused by podocyte damage is associated with the inherited and acquired impairment of autophagic clearance and lysosome depletion. In Fabry's disease, lysosomal enzymatic deficiency resulted from a mutation in the gene encoding a-galactoside A causes dysregulation of autophagic flux and contributes to podocyte damage, leading to the occurrence of proteinuria [59]. Podocyte-specific deletion of lysosomal acid ceramidase causes effacement of foot processes and severe proteinuria in mice. Moreover, loss of lysosome cathepsin D in podocytes is associated with the accumulation of abundant autophagosomes/autolysosome-like bodies which trigger the apoptotic podocyte death followed by proteinuria and glomerulosclerosis [60]. In contrast, the induction of cathepsin L expression in podocytes may mediate the development of proteinuria in puromycin aminonucleoside nephropathy. We also find that the complement membrane attack complex can induce podocyte injury in idiopathic membranous nephropathy by triggering LMP and blocking autophagic flux [61]. The function of Janus kinase 2 (JAK2) in podocytes is to maintain autophagy completion, and mice with JAK2 deficiency in podocytes exhibit increased urinary albumin excretion, accompanied by autophagosome accumulation and p62 aggregation [62].

Proteins filtered through the glomerular filtration barrier can be reabsorbed by TECs, followed by digestion and reuse. Upregulation of lysosomal proteolysis in TECs is required to maintain the normal clearance of megalin/ tubulin-mediated reabsorbed proteins. Once the urinary protein is overload, the degradation of lysosomes in proximal tubules occurs, which results in tubulointerstitial inflammation and fibrosis via various mechanisms, including oxidative stress, mitochondrial dysfunction, and ER stress. In our previous study, we found that activation of autophagy through removal of damaged mitochondria attenuates TEC injury induced by overload of urinary proteins from patients $[63,64]$ However, after long-term exposure to overload of urinary proteins, excess oxidative stress is caused by LMP and lysosomal dysfunction in TECs, resulting in blockage of autophagic flux [65]. Nolin et al. [66] also found that albumin overload suppresses proximal tubule autophagy in an mTOR-mediated mechanism. Recently, Liu et al. $[67,68]$ showed that albumin overload causes lysosome rupture and defects in autophagic flux. Additionally, LMP releases lysosomal hydrolases, which subsequently triggers activation of the NLRP3 inflammasome in TECs.

\section{Acute Kidney Injury}

Acute kidney injury (AKI), characterized by a rapid loss of renal function, is a global public health concern associated with high morbidity, mortality, and healthcare costs [69]. AKI can be caused by various conditions including ischemia-reperfusion injury, sepsis, and exposure to nephrotoxins, generally resulting in sublethal and lethal damage to renal tubules. Additionally, patients with AKI are more likely to develop into CKD, and the process is largely determined by whether tubular cells recover sufficiently [69].

Autophagy plays protective role in tubular cells in AKI. Proximal tubule-specific depletion of ATG7 or ATG5 in mice is more sensitive to renal injury in response to AKI both induced by cisplatin and ischemia-reperfusion [70]. In contrast, overexpression of ATG5 and beclin-1 inhibits the activation of caspase and cell death, protecting renal tubular cells from cisplatin toxicity [71]. Furthermore, we found that selective autophagic processes are impaired in sepsis-induced AKI, leading to the accumulation of damaged mitochondria, increased oxidative stress, and enhanced tubular cell death [72].

Some studies have shown that the impairment of autophagic clearance is also involved in AKI. For example, disruption of autophagic clearance by the lysosome inhibitor chloroquine worsens cisplatin nephrotoxicity [70]. Aged mice sensitive to AKI exhibit weakening of autophagic clearance in tubular cells. In addition, autophagic clearance may contribute to the recovery of tubular cells after AKI. Lin's group showed that the process of renal recovery is accompanied by the initiation of autophagy and the clearance of autophagosome in autophagic reporter mice. They also reported that tubular cells with autophagosome accumulation are little proliferative with delayed tubular repair [73]. Moreover, blockage of autophagic flux by the lysosome inhibitor, chloroquine or bafilomycin A, promotes G1-phase cell cycle arrest in- 
duced by transforming growth factor- $\beta$ in renal TECs [74]. Thus, impaired autophagic clearance and lysosome depletion may promote tubular injury and delay tubular repair.

\section{Crystalline Nephropathy}

Kidney is the predominant organ for crystal deposition. The concentrations of mineral secretion and supersaturation in urine can cause the diverse crystal nephropathies and kidney stone diseases. Crystals not only obstruct the tubular lumen but also are widely taken up by TECs and even reach the tubulointerstitium. Although the formation and types of crystals vary, the pathological mechanisms of crystal-induced renal damage seem similar. Crystals can be engulfed into the phagosomes by tubular cells and delivered to fuse with lysosomes for digestion with lysosomal proteolytic enzymes. Because it is impossible to digest these crystals, they can destabilize the lysosomal membrane and induce LMP. Leakage of lysosomal proteolytic enzymes (such as cathepsin B) from the lysosome into the cytoplasm triggers ROS production, NLRP3 inflammasome activation, or RIPK1/RIPK3/phospho-MLKL necroptosis activation, resulting in tubular cell death and tubulointerstitial inflammation [75]. In response to calcium oxalate monohydrate crystals, the autophagy activity is markedly decreased in mouse renal tubular cells, accompanied by accumulation of damaged mitochondria and lysosomes. mTOR-mediated TFEB suppression contributes to the impairment of autophagic activity [76]. In uric acid nephropathy, uric acid crystals elevate the levels of urate transporter 1 via suppression of Numb-mediated lysosome-dependent degradation [77]. Thus, lysosome depletion and autophagic clearance impairment are also involved in many types of crystalline nephropathy.

\section{Drug- and Heavy Metal-Induced Renal Damage}

Many clinically used drugs are associated with tubular cell toxicity. These drugs induce tubule injury through various mechanisms, such as mitochondrial dysfunction, oxidative stress, lysosome depletion, and autophagic clearance impairment. For example, the aminoglycoside antibiotic gentamicin accumulates in lysosomes and induces ROS-mediated LMP, which subsequently triggers mitochondrial-dependent apoptosis in TECs $[78,79]$. Autophagy protects against renal damage induced by the immunosuppressor cyclosporine A (CsA) [80]. However, chronic administration of CsA causes renal injury associated with increased autophagosome formation and reduced autophagosome clearance [80], which may be attributed to the lysosome depletion [81]. During CsA-in- duced autophagy in TECs, the transmembrane bax inhibitor motif containing 6 plays key roles in maintaining autophagic flux via activation of lysosomes in vitro and in vivo [82]. The lysosome inhibitor hydroxychloroquine is recommended for the management of autoimmune diseases, including lupus, and has been shown to protect against AKI in mice by suppressing NLRP3 inflammasome activation [83]. However, in rare cases, hydroxychloroquine accumulates in lysosomes and induces cytoplasmic vacuolization and zebra bodies in podocytes, which are mimicking the histological lesions of Fabry's disease and ultimately resulting in podocyte injury and proteinuria $[84,85]$.

Heavy metal-induced tubular injury is also related to the autophagy-lysosome pathway. Mercuric chloride impairs lysosome function in tubular cells [86]. Additionally, lead promotes autophagosome accumulation in primary rat proximal tubular cells by inducing lysosome alkalinization via inhibition of $2 \mathrm{~V}$-ATPase subunits of lysosomes, rather than through suppressing autophagosome-lysosome fusion. Moreover, blockage of autophagic flux and LMP contributes to caspase-3-mediated apoptosis in lead-treated tubular cells [87]. In cadmium nephrotoxicity, autophagy protects against tubular injury under low cadmium stress. However, high cadmium stress disrupts lysosome stability and impairs autophagic flux, leading to cell death [88].

As mentioned above, all the pathogenic factors (including AGEs, high glucose, proteinuric, crystalline, drug, and heavy metal and so on) could impair autophagic flux, trigger inflammasome activation, and cause cell death through disrupting the integrity of lysosomal membrane and promoting the leakage of lysosomal proteolytic enzymes. That might be one of the key underlying mechanisms to induce kidney injury and promote the progression of CKD. The relationship between lysosome depletion and these kidney diseases are summarized in Table 1.

\section{Potential Therapeutic Strategies for Management of Kidney Diseases by Targeting Lysosome Depletion-Caused Autophagic Clearance Impairment}

To mitigate lysosome depletion and autophagic clearance impairment, cells can develop multiple protective mechanisms, including the rescue of slightly damaged lysosomes, the removal of severely damaged lysosomes, and the regeneration of new lysosomes, which is ultimately promoting autophagic clearance and cell survival. 
Table 1. The part of autophagy disruption in different type of kidney diseases

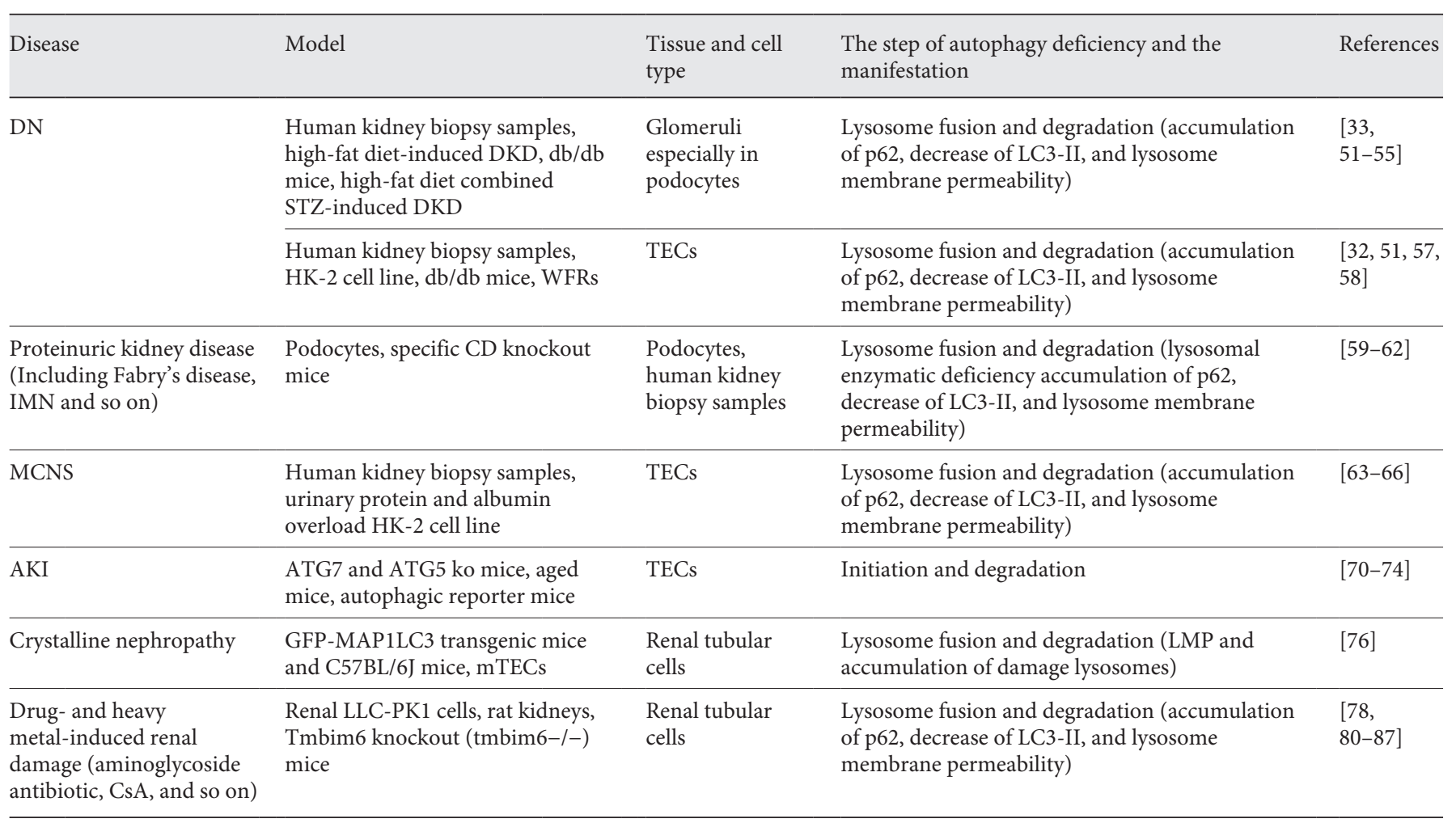

DN, diabetic nephropathy; TECs, tubular epithelial cells; AKI, acute kidney injury; CsA, cyclosporine A; WFRs, Wistar fatty (fa/fa) rats; LMP, lysosomal membrane permeabilization.

\section{Rescue of Lysosomes Mediated by the Endosomal}

Sorting Complex Required for Transport

If lysosome damage is limited, it can be repaired by the endosomal sorting complex required for transport (ESCRT) machinery. ESCRT is an evolutionarily conserved membrane remodeling process that is involved in endosomal trafficking and is essential for generating vesicles that bud into multivesicular endosomes [89]. The ESCRT machinery consists of 5 distinct protein complexes (ESCRT-0, I, II, and III and the Vps4 complex) that transiently assemble a multisubunit complex via a topologically unique membrane bending and scission reaction away from the cytoplasm. The modular setup of this machinery enables flexible integration into very different 3 biological processes from the interaction with ubiquitinated membrane proteins to the membrane deformation and abscission [90].

Consistent with the results reported in a recent study [89], Radulovic and colleagues found that ESCRT can mediate the repair of damaged lysosomes. Besides, they found that ESCRT-mediated membrane repair provides an advantage effect in promoting cell viability after lysosome injury. This mechanism can also be involved in the maintenance of intact replicative niche for intracellular pathogens [91]. ESCRT drives membrane remodeling prior to fission and plays distinct but equally important roles in the downregulation of surface transporter and receptor mediated by the multivesicular body pathway [92]. Unfortunately, no studies have yet shown whether ESCRTs have protective roles in kidney diseases.

\section{Removal of Damaged Lysosomes via Promotion of Lysophagy}

Presumably, if ESCRT-mediated membrane repair fails, these damaged lysosomes are then selectively eliminated by autophagic degradation termed lysophagy. Lysophagy is an essential process for maintaining intracellular homeostasis $[93,94]$, which is initiated by ubiquitination of lysosomal proteins [95]. After membrane damage, the ubiquitination occurs, which is 30 -min delay compared to the recruitment of the ESCRT components. This observation indicates that unrepairable lysosomes 
eliminated by lysophagy is independent on ESCRT-mediated repair process. Unlike the ESCRT machinery that is recruited by the subtler cues, the initiation of lysophagy requires holes large enough in the severely damaged lysosomalmembranefor detectingintraluminal $\beta$-galactosides via cytosolic lectins such as galectin-3 [91]. When severer LMP occurs, galectin-3 binds to lysosomal glycans and recruits the atypical tripartite motif (TRIM) family protein TRIM16. It promotes a robust ubiquitination of the damaged lysosome and formation of a platform for the autophagic machinery, including ULK1, ATG16L, and beclin-1, to initiate selective autophagy [93]. Chrisovalantis et al. [94] found that damaged lysosomes are selectively ubiquitinated and are degraded eventually by incorporate into autolysosomes. Analysis of lysosome damage induced by LLOMe shows that the recruitment of ubiquitination is as importance as autophagy receptor p62 for maintaining efficiency of lysophagy. It has identified that the E2 enzyme UBE2QL1 is involved in catalyzing the ubiquitination of damaged lysosomes [95]. Without this enzyme, the process of removing the ruptured lysosomes by lysophagy is compromised both upon lysosome damage and under normal conditions [96-98]. Thus, lysophagy represents a crucial mechanism of lysosomal quality control [94].

The protective role of lysophagy in kidney disease has mainly been reported in crystalline nephropathy. Notably, inhibition of mTOR promotes the activity of the autophagy pathway and suppresses crystal-induced inflammatory responses in tubular cells. Moreover, lysosomes damaged by urate crystals can be sequestered, and the decreased degradation ability of lysosomes can be repaired in an autophagy-dependent manner (lysophagy). In addition, autophagy not only mediates the clearance of damaged lysosomes but also promotes lysosome biogenesis [99].

\section{TFEB-Mediated Lysosome Biogenesis}

If there is an overload of damaged lysosomes, lysophagy cannot clear the damaged lysosomes due to deficiencies in functional lysosomes. Subsequently, lysosome biogenesis is initiated to overcome this lysosome depletion. In recent studies regarding lysosome biogenesis, TFEB, a member of the microphthalmia family of basic helixloop-helix leucine-zipper transcription factors, has received a lot of attention [100]. TFEB mediates lysosome biogenesis by directly binding to the promoter sequences containing the coordinated lysosomal expression and regulation element, thereby initiating the transcription of its target gene network encoding lysosome, endosome, and autophagy proteins. Thus, TFEB activation caused by stress can drive lysosome biogenesis and autophagic flux to adapt and scale-up the activity of the endo-lysosomal system [101].

The most widely studied responsible regulation of TFEB activation is mTOR-mediated phosphorylation, which is closely relevant to the functional status of its resident organelle, the lysosome. Phosphorylated TFEB is sequestered into the cytoplasm in the presence of nutrients, whereas dephosphorylated TFEB rapidly translocates from the cytoplasm to the nucleus under conditions of starvation or lysosome dysfunction. Within the nucleus, it binds to the coordinated lysosomal expression and regulation element and consequently promotes the transcriptional activation of downstream target genes $[102,103]$. Recently, advances in the regulation of TFEB have demonstrated the unexpected complexity of this system. Sha et al. [104] found that inactive phosphorylated TFEB is preferentially ubiquitylated by the E3 ubiquitin ligase STUB1 for proteasomal degradation; however, dephosphorylated TFEB is spared from the ubiquitylation of STUB1 and accumulates in the nucleus. Consequently, increased phosphorylated TFEB in STUB1-deficient cells further compromises the TFEB activity by forming heterodimers with dephosphorylated TFEB and inhibits the translocation of them to the nucleus even in a state of starvation. Thus, TFEB activity is mediated by STUB1 through promoting the proteasomal degradation of inactive TFEB and reducing the formation of heterodimerization [101]. Pan et al. [105] found that, in cardiac proteinopathy, activation of mTORC1 impaired TFEB signaling. However, overexpression of TFEB protects against proteotoxicity in cardiomyocytes through improving autophagy-lysosome pathway activity.

Emerging evidence has shown that promotion of TFEB-mediated lysosome biogenesis attenuates renal injury [106]. Zhao et al. [107] found that hyperactivity of mTOR inhibits TFEB-mediated lysosome biogenesis and autophagy, thereby suppressing autophagic flux in diabetic podocytes. Additionally, suppression of mTOR activity recovers TFEB nuclear translocation and prevents AGE-induced autophagy insufficiency. Furthermore, we showed that restoration of lysosomes by resveratrol plus vitamin E treatment rescues autophagic flux to alleviate podocyte injury in $\mathrm{DN}$ [33]. We also found that decreased TFEB expression impairs lysosome biogenesis and autophagic flux in TECs in DN [58]. In contrast, enhanced TFEB activity promotes lysosome biogenesis and digestive function, resulting in suppression of tubular cell in- 


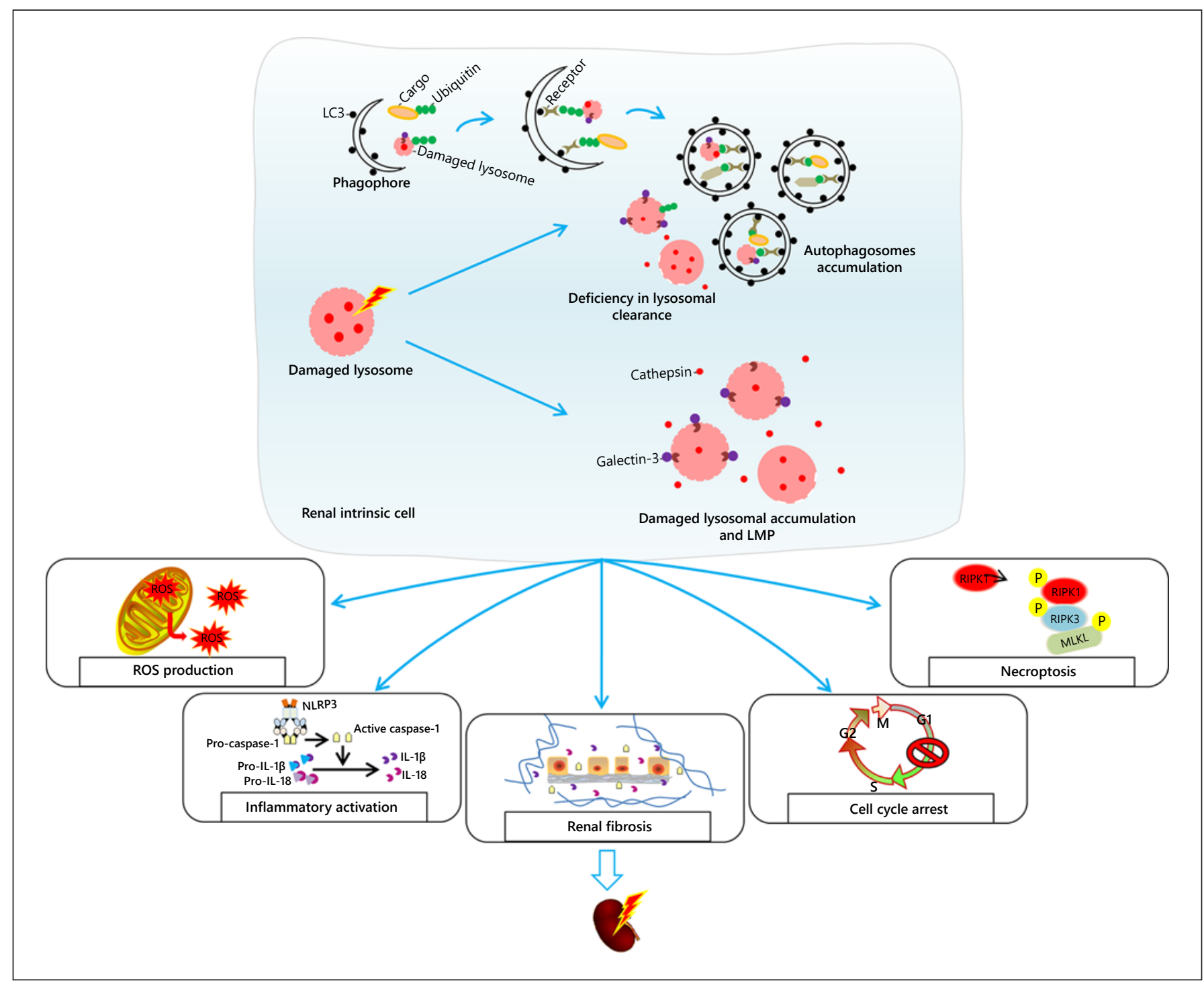

Fig. 1. Potential mechanisms of renal injury triggered by lysosome depletion in kidney diseases. Lysosome rupture that causes lysosomal dysfunction and the release of endogenous hydrolytic enzymes, leading to the disruption of autophagic flux and LMP, which further triggers ROS production, NLRP3 inflammasome activation, cell cycle arrest, and RIPK1/RIPK3/phospho-MLKL-me-

jury in vitro and in vivo. In proteinuric kidney disease, deletion of JAK2 downregulates TFEB expression, whereas TFEB overexpression enhances lysosome function and rescues autophagic flux, resulting in improvement of podocyte function [62]. Recently, we found that rescue of lysosome depletion via activation of TFEB-mediated lysosome biogenesis repairs blockage of autophagic flux and suppresses apoptosis in TECs stimulated by urinary proteins. In addition, we found that $\operatorname{Smad} 3$ participates in the diated necroptosis in renal intrinsic cells, consequently promoting renal fibrosis and injury. LC3, microtubule-associated protein 1 light chain 3; LMP, lysosomal membrane permeabilization; ROS, reactive oxygen species; IL- $1 \beta$, interleukin- $1 \beta$; IL-18, interleukin-18.

progression of DN by downregulation of TFEB, and inhibition of Smad3 enhanced the expression of TFEB [58]. Additionally, stimulation of endogenous TFEB activity or overexpression of exogenous TFEB also rescues lysosome abnormalities related to cystinosin deficiency in TECs [108]. Overall, enhancing autophagic flux by TFEB-mediated lysosome biogenesis can suppress renal damage in many kidney diseases. 


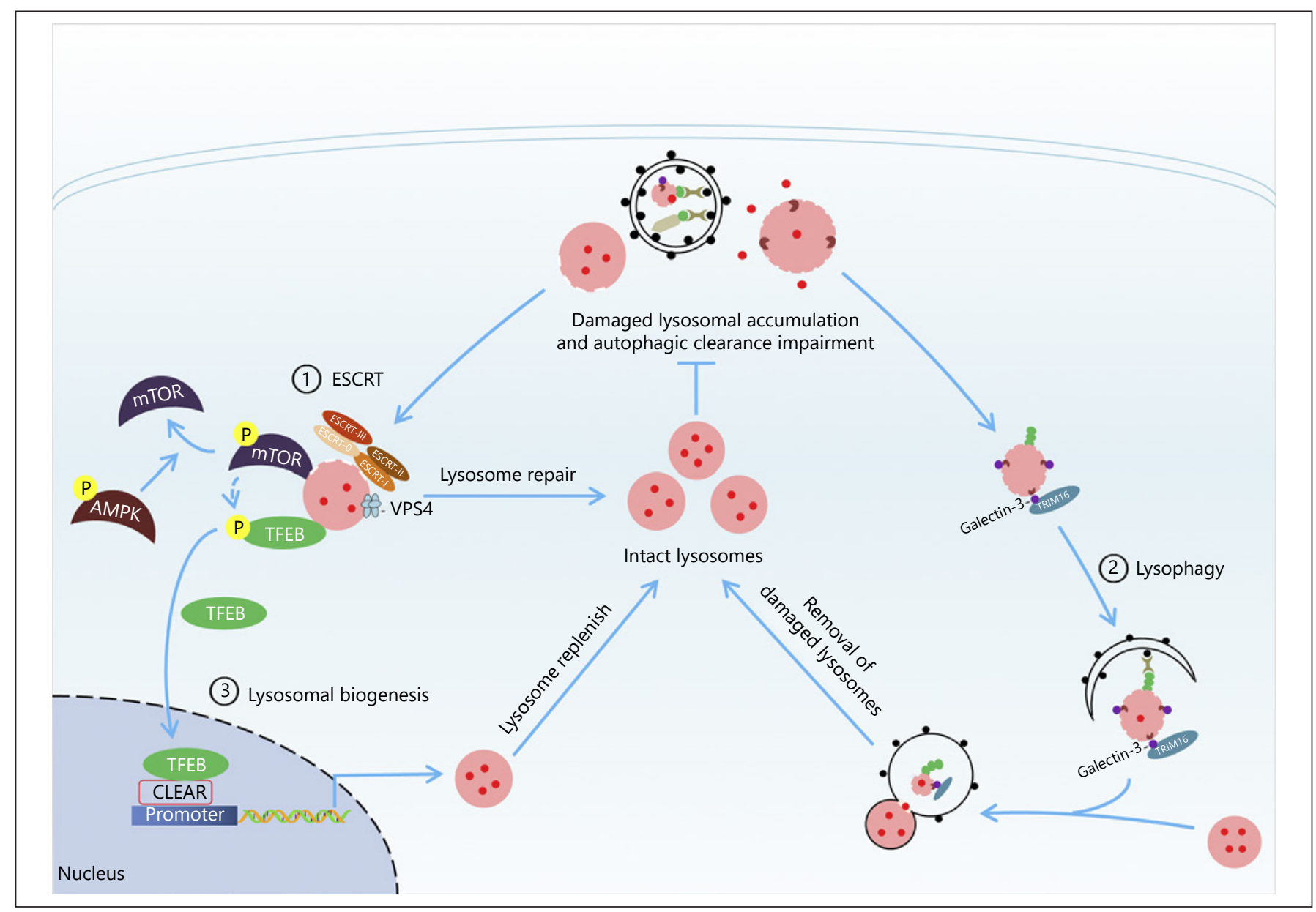

Fig. 2. Overview of potential protective mechanisms for coping with the lysosome depletion. Different repair mechanisms have been discovered to restore the function of lysosome depending on the extent of lysosomal damage, mainly through 3 potential mechanisms: the rescue of slightly damaged lysosomes mediated by ESCRT machinery, the removal of severely damaged lysosomes via lysophagy, and lysosome biogenesis regulated by TFEB that coor-

\section{Drugs Targeting Lysosome Depletion}

Because the mechanisms of ESCRT-mediated lysosome repair and lysophagy are not fully elucidated, there are currently no drugs for selectively targeting them to rescue lysosome. Only lysosome biogenesis by targeting TFEB has been utilized in some diseases including kidney diseases. Trehalose was confirmed to promote autophagy and attenuate cisplatin-induced AKI through activating TFEB [109]. Urolithin A protects against ischemia/reperfusion-induced renal injury in mice by enhancing TFEBmediated autophagy [110]. Zhang et al. [111] found that curcumin has a potential anticancer function by inhibiting $\mathrm{mTOR}$ and promoting TFEB nuclear translocation. dinately replenish intact lysosomes for restoring lysosome function and improving autophagic clearance, which may provide a potential therapy strategies for the management of kidney diseases. ESCRT, the endosomal sorting complex required for transport; mTOR, mammalian target of rapamycin; AMPK, AMP-activated protein kinase; TFEB, transcription factor $\mathrm{EB}$.

Moskot et al. [112] also found that the beneficial effect of genistein in lysosomal storage diseases is attributed to genistein-mediated enhancement of TFEB gene expression, TFEB nuclear translocation, and activation of TFEB-dependent lysosome biogenesis to lysosomal metabolism. In addition, the mTOR inhibitors such as Torin1 [113], pp242 [114], rapamycin [115], naringenin, and the drugs used for anticancer treatment such as doxorubicin [116, 117] and mitoxantrone [116] can also increase the expression of TFEB and promote its nuclear translocation. All the drugs we mentioned above might be potential therapeutic agent for kidney diseases caused by lysosomes depletion. 


\section{Conclusion}

Autophagic clearance impairment caused by lysosome depletion is tightly associated with many types of kidney diseases. Lysosome depletion induces NLRP3 activation, ROS production, RIPK1/RIPK3/phospho-MLKL-mediated necroptosis, cell cycle arrest, and growth suppression in renal intrinsic cells, promoting the progression of renal inflammation and fibrosis (Fig. 1). In addition, rescue of lysosomes mediated by ESCRT, removal of damaged lysosomes via lysophagy, and lysosome biogenesis mediated by TFEB can restore lysosome function and improve autophagic clearance (Fig. 2). Thus, better understanding of the regulatory mechanisms involving in the lysosome function and autophagy pathway may be the first step toward the development of novel therapeutic strategies for kidney diseases.

\section{Acknowledgement}

There are no acknowledgments to declare.

\section{Conflict of Interest Statement}

No potential conflicts of interest were disclosed.

\section{Funding Sources}

This work was supported by grants from the National Natural Science Foundation of China (No. 81670654, No. 81700627, and No. 81974095); the Funds for Science and Technology Innovation Strategy of Guangdong Province (2019A1515010678); the Grants Research Council of Hong Kong (GRF 14163317, 14117418, 14104019, C7018-16G, and R4012-18), and the Health and Medical Research Fund of Hong Kong (05161326 and 14152321); and the Guangdong-Hong Kong-Macao-Joint Labs Program from Guangdong Science and Technology Department (2019B121205005).

\section{Author Contributions}

X.C.C., Z.H.L., and C.Y. wrote the draft of the review. J.X.T. helped to revise the review. H.F.L. and H.Y.L. designed and approved this work.

\section{References}

1 Mizushima N, Levine B, Cuervo AM, Klionsky DJ. Autophagy fights disease through cellular self-digestion. Nature. 2008 Feb 28; 451(7182):1069-75.

2 Murrow L, Debnath J. Autophagy as a stressresponse and quality-control mechanism: implications for cell injury and human disease. Annu Rev Pathol. 2013 Jan 24;8:105-37.

3 Pyo JO, Nah J, Jung YK. Molecules and their functions in autophagy. Exp Mol Med. 2012 Feb 29;44(2):73-80.

4 Sureshbabu A, Ryter SW, Choi ME. Oxidative stress and autophagy: crucial modulators of kidney injury. Redox Biol. 2015;4:208-14.

5 Zhang L, Sheng R, Qin Z. The lysosome and neurodegenerative diseases. Acta Biochim Biophys Sin. 2009 Jun;41(6):437-45.

6 Wu Y, Yao J, Xie J, Liu Z, Zhou Y, Pan H, et al. The role of autophagy in colitis-associated colorectal cancer. Signal Transduct Target Ther. 2018;3:31.

7 Wang J, Davis S, Zhu M, Miller EA, FerroNovick S. Autophagosome formation: where the secretory and autophagy pathways meet. Autophagy. 2017 May 4;13(5):973-4.

$8 \mathrm{He}$ C, Klionsky DJ. Regulation mechanisms and signaling pathways of autophagy. Annu Rev Genet. 2009;43:67-93.

9 Hosseinpour-Moghaddam K, Caraglia M, Sahebkar A. Autophagy induction by trehalose: molecular mechanisms and therapeutic impacts. J Cell Physiol. 2018 Sep;233(9):652443.
10 Tavakol S, Ashrafizadeh M, Deng S, Azarian M, Abdoli A, Motavaf M, et al. Autophagy modulators: mechanistic aspects and drug delivery systems. Biomolecules. 2019 Sep 25; 9(10):530

11 Shou J, Wang M, Cheng X, Wang X, Zhang L, Liu Y, et al. Tizoxanide induces autophagy by inhibiting $\mathrm{PI} 3 \mathrm{~K} / \mathrm{Akt} / \mathrm{mTOR}$ pathway in RAW264.7 macrophage cells. Arch Pharm Res. 2020 Feb;43(2):257-70.

12 Civiletto G, Dogan SA, Cerutti R, Fagiolari G, Moggio M, Lamperti C, et al. Rapamycin rescues mitochondrial myopathy via coordinated activation of autophagy and lysosomal biogenesis. EMBO Mol Med. 2018 Nov;10(11): e8799.

13 Liu J, Long S, Wang H, Liu N, Zhang C, Zhang $\mathrm{L}$, et al. Blocking AMPK/ULK1-dependent autophagy promoted apoptosis and suppressed colon cancer growth. Cancer Cell Int. 2019;19:336.

14 Narendra DP, Youle RJ. Targeting mitochondrial dysfunction: role for PINK1 and Parkin in mitochondrial quality control. Antioxid Redox Signal. 2011 May 15;14(10):1929-38.

15 Chen RH, Chen YH, Huang TY. Ubiquitinmediated regulation of autophagy. J Biomed Sci. 2019 Oct 21;26(1):80.

16 Nakamura S, Yoshimori T. New insights into autophagosome-lysosome fusion. J Cell Sci. 2017 Apr 1;130(7):1209-16.
17 Cabukusta B, Neefjes J. Mechanisms of lysosomal positioning and movement. Traffic. 2018 Oct;19(10):761-9.

18 Kabeya Y, Mizushima N, Ueno T, Yamamoto A, Kirisako T, Noda T, et al. LC3, a mammalian homologue of yeast Apg8p, is localized in autophagosome membranes after processing. EMBO J. 2000 Nov 1;19(21):5720-8.

19 Korolchuk VI, Rubinsztein DC. Regulation of autophagy by lysosomal positioning. Autophagy. 2011 Aug;7(8):927-8.

20 Lorincz P, Juhasz G. Autophagosome-lysosome fusion. J Mol Biol. 2020 Apr 3;432(8): 2462-82.

21 Perera RM, Zoncu R. The lysosome as a regulatory hub. Annu Rev Cell Dev Biol. 2016 Oct 6;32:223-53

22 de Araujo MEG, Liebscher G, Hess MW, Huber LA. Lysosomal size matters. Traffic. 2020 Jan;21(1):60-75.

23 Wang F, Gómez-Sintes R, Boya P. Lysosomal membrane permeabilization and cell death. Traffic. 2018 Dec;19(12):918-31.

24 Ballabio A. The awesome lysosome. EMBO Mol Med. 2016 Feb 1;8(2):73-6.

25 Friedman LG, Qureshi YH, Yu WH. Promoting autophagic clearance: viable therapeutic targets in Alzheimer's disease. Neurotherapeutics. 2015 Jan;12(1):94-108.

26 Yang C, Wang X. Cell biology in China: focusing on the lysosome. Traffic. 2017 Jun;18(6): $348-57$. 
27 Yim WW, Mizushima N. Lysosome biology in autophagy. Cell Discov. 2020;6(1):6.

28 Ma X, Liu H, Foyil SR, Godar RJ, Weinheimer CJ, Hill JA, et al. Impaired autophagosome clearance contributes to cardiomyocyte death in ischemia/reperfusion injury. Circulation. 2012 Jun 26;125(25):3170-81.

29 Sarkar C, Zhao Z, Aungst S, Sabirzhanov B, Faden AI, Lipinski MM. Impaired autophagy flux is associated with neuronal cell death after traumatic brain injury. Autophagy. 2014; 10(12):2208-22.

30 Cui D, Sun D, Wang X, Yi L, Kulikowicz E, Reyes $\mathrm{M}$, et al. Impaired autophagosome clearance contributes to neuronal death in a piglet model of neonatal hypoxic-ischemic encephalopathy. Cell Death Dis. 2017 Jul 13; 8(7):e2919.

31 Wang J, Yu Y, Lu K, Yang M, Li Y, Zhou X, et al. Silica nanoparticles induce autophagy dysfunction via lysosomal impairment and inhibition of autophagosome degradation in hepatocytes. Int J Nanomed. 2017;12:809-25.

32 Liu WJ, Shen TT, Chen RH, Wu HL, Wang YJ, Deng JK, et al. Autophagy-lysosome pathway in renal tubular epithelial cells is disrupted by advanced glycation end products in diabetic nephropathy. J Biol Chem. 2015 Aug 14; 290(33):20499-510.

33 Liu WJ, Gan Y, Huang WF, Wu HL, Zhang $\mathrm{XQ}$, Zheng HJ, et al. Lysosome restoration to activate podocyte autophagy: a new therapeutic strategy for diabetic kidney disease. Cell Death Dis. 2019 Oct 24;10(11):806.

34 Boya P, Kroemer G. Lysosomal membrane permeabilization in cell death. Oncogene. 2008 Oct 27;27(50):6434-51.

35 Yu F, Chen Z, Wang B, Jin Z, Hou Y, Ma S, et al. The role of lysosome in cell death regulation. Tumour Biol. 2016 Feb;37(2):1427-36.

36 Serrano-Puebla A, Boya P. Lysosomal membrane permeabilization in cell death: new evidence and implications for health and disease. Ann N Y Acad Sci. 2016 May;1371(1):30-44.

37 Fehrenbacher N, Bastholm L, Kirkegaard-Sørensen T, Rafn B, Bøttzauw T, Nielsen C, et al. Sensitization to the lysosomal cell death pathway by oncogene-induced down-regulation of lysosome-associated membrane proteins 1 and 2. Cancer Res. 2008 Aug 15;68(16):662333.

38 Ono K, Kim SO, Han J. Susceptibility of lysosomes to rupture is a determinant for plasma membrane disruption in tumor necrosis factor alpha-induced cell death. Mol Cell Biol. 2003 Jan;23(2):665-76.

39 Chen W, Li N, Chen T, Han Y, Li C, Wang Y, et al. The lysosome-associated apoptosis-inducing protein containing the pleckstrin homology (PH) and FYVE domains (LAPF), representative of a novel family of $\mathrm{PH}$ and FYVE domain-containing proteins, induces caspase-independent apoptosis via the lysosomal-mitochondrial pathway. J Biol Chem. 2005 Dec 9;280(49):40985-95.
40 Li N, Zheng Y, Chen W, Wang C, Liu X, He $\mathrm{W}$, et al. Adaptor protein LAPF recruits phosphorylated p53 to lysosomes and triggers lysosomal destabilization in apoptosis. Cancer Res. 2007 Dec 1;67(23):11176-85.

41 Kreuzaler PA, Staniszewska AD, Li W, Omid$\operatorname{var}$ N, Kedjouar B, Turkson J, et al. Stat3 controls lysosomal-mediated cell death in vivo. Nat Cell Biol. 2011 Mar;13(3):303-9.

42 Guicciardi ME, Deussing J, Miyoshi H, Bronk SF, Svingen PA, Peters C, et al. Cathepsin B contributes to TNF-alpha-mediated hepatocyte apoptosis by promoting mitochondrial release of cytochrome c. J Clin Invest. 2000 Nov; 106(9):1127-37.

43 Werneburg NW, Guicciardi ME, Bronk SF, Gores GJ. Tumor necrosis factor-alpha-associated lysosomal permeabilization is cathepsin B dependent. Am J Physiol Gastrointest Liver Physiol. 2002 Oct;283(4):G947-56.

44 Liu N, Raja SM, Zazzeroni F, Metkar SS, Shah $\mathrm{R}$, Zhang M, et al. NF-kappaB protects from the lysosomal pathway of cell death. EMBO J. 2003 Oct 1;22(19):5313-22.

45 Hartleben B, Gödel M, Meyer-Schwesinger C, Liu S, Ulrich T, Köbler S, et al. Autophagy influences glomerular disease susceptibility and maintains podocyte homeostasis in aging mice. J Clin Invest. 2010 Apr;120(4):1084-96.

46 Kume S, Uzu T, Horiike K, Chin-Kanasaki M, Isshiki K, Araki S, et al. Calorie restriction enhances cell adaptation to hypoxia through Sirt1-dependent mitochondrial autophagy in mouse aged kidney. J Clin Invest. 2010 Apr; 120(4):1043-55.

47 Fiseha T. Urinary biomarkers for early diabetic nephropathy in type 2 diabetic patients. Biomark Res. 2015;3:16.

48 Johnson SA, Spurney RF. Twenty years after ACEIs and ARBs: emerging treatment strategies for diabetic nephropathy. Am J Physiol Renal Physiol. 2015 Nov 15;309(10):F807-20.

49 Liu J, Wang C, Liu F, Lu Y, Cheng J. Metabonomics revealed xanthine oxidase-induced oxidative stress and inflammation in the pathogenesis of diabetic nephropathy. Anal Bioanal Chem. 2015 Mar;407(9):2569-79.

50 Zhang X, He H, Liang D, Jiang Y, Liang W, $\mathrm{Chi} \mathrm{ZH}$, et al. Protective effects of berberine on renal injury in streptozotocin (STZ)-induced diabetic mice. Int J Mol Sci. 2016 Aug 12;17(8):1327.

51 Kitada M, Ogura Y, Monno I, Koya D. Regulating autophagy as a therapeutic target for diabetic nephropathy. Curr Diab Rep. 2017 Jul;17(7):53.

52 Tagawa A, Yasuda M, Kume S, Yamahara K, Nakazawa J, Chin-Kanasaki M, et al. Impaired podocyte autophagy exacerbates proteinuria in diabetic nephropathy. Diabetes. 2016 Mar;65(3):755-67.

53 Terami N, Ogawa D, Tachibana H, Hatanaka T, Wada J, Nakatsuka A, et al. Long-term treatment with the sodium glucose cotransporter 2 inhibitor, dapagliflozin, ameliorates glucose homeostasis and diabetic nephropathy in $\mathrm{db} /$ db mice. PLoS One. 2014;9(6):e100777.
54 Wang X, Gao L, Lin H, Song J, Wang J, Yin Y, et al. Mangiferin prevents diabetic nephropathy progression and protects podocyte function via autophagy in diabetic rat glomeruli. Eur J Pharmacol. 2018 Apr 5;824:170-8.

55 Dong D, Fan TT, Ji YS, Yu JY, Wu S, Zhang L. Spironolactone alleviates diabetic nephropathy through promoting autophagy in podocytes. Int Urol Nephrol. 2019 Apr;51(4): 755-64.

56 Takahashi A, Takabatake Y, Kimura T, Maejima I, Namba T, Yamamoto T, et al. Autophagy inhibits the accumulation of advanced glycation end products by promoting lysosomal biogenesis and function in the kidney proximal tubules. Diabetes. 2017 May;66(5): 1359-72.

57 Kitada M, Takeda A, Nagai T, Ito H, Kanasaki K, Koya D. Dietary restriction ameliorates diabetic nephropathy through anti-inflammatory effects and regulation of the autophagy via restoration of Sirtl in diabetic Wistar fatty (fa/fa) rats: a model of type 2 diabetes. Exp Diabetes Res. 2011;2011:908185.

58 Yang C, Chen X-C, Li Z-H, Wu H-L, Jing K-P, Huang X-R, et al. SMAD3 promotes autophagy dysregulation by triggering lysosome depletion in tubular epithelial cells in diabetic nephropathy. Autophagy. 2020 Oct 12:1-20.

59 Liebau MC, Braun F, Höpker K, Weitbrecht C, Bartels V, Müller RU, et al. Dysregulated autophagy contributes to podocyte damage in Fabry's disease. PLoS One. 2013;8(5):e63506.

60 Yamamoto-Nonaka K, Koike M, Asanuma K, Takagi M, Oliva Trejo JA, Seki T, et al. Cathepsin $\mathrm{D}$ in podocytes is important in the pathogenesis of proteinuria and CKD. J Am Soc Nephrol. 2016 Sep;27(9):2685-700.

61 Liu WJ, Li ZH, Chen XC, Zhao XL, Zhong Z, Yang $\mathrm{C}$, et al. Blockage of the lysosome-dependent autophagic pathway contributes to complement membrane attack complex-induced podocyte injury in idiopathic membranous nephropathy. Sci Rep. 2017 Aug 17;7(1): 8643.

62 Alghamdi TA, Majumder S, Thieme K, Batchu SN, White KE, Liu Y, et al. Janus kinase 2 regulates transcription factor EB expression and autophagy completion in glomerular podocytes. J Am Soc Nephrol. 2017 Sep;28(9):2641-53.

63 Liu WJ, Luo MN, Tan J, Chen W, Huang LZ, Yang C, et al. Autophagy activation reduces renal tubular injury induced by urinary proteins. Autophagy. 2014 Feb;10(2):243-56.

64 Tan J, Wang M, Song S, Miao Y, Zhang Q. Autophagy activation promotes removal of damaged mitochondria and protects against renal tubular injury induced by albumin overload. Histol Histopathol. 2018 Jul;33(7):68190.

65 Liu WJ, Xu BH, Ye L, Liang D, Wu HL, Zheng YY, et al. Urinary proteins induce lysosomal membrane permeabilization and lysosomal dysfunction in renal tubular epithelial cells. Am J Physiol Renal Physiol. 2015 Mar 15; 308(6):F639-49. 
66 Nolin AC, Mulhern RM, Panchenko MV, Pisarek-Horowitz A, Wang Z, Shirihai O, et al. Proteinuria causes dysfunctional autophagy in the proximal tubule. Am J Physiol Renal Physiol. 2016 Dec 1;311(6):F1271-F79.

67 Liu D, Wen Y, Tang TT, Lv LL, Tang RN, Liu $\mathrm{H}$, et al. Megalin/cubulin-lysosome-mediated albumin reabsorption is involved in the tubular cell activation of NLRP3 inflammasome and tubulointerstitial inflammation. J Biol Chem. 2015 Jul 17;290(29):18018-28.

68 Liu L, Li Y, Wang Z, Ding F, Cheng Z, Xu Q, et al. Rab7 empowers renal tubular epithelial cells with autophagy-mediated protection against albumin-induced injury. Exp Cell Res. 2018 Sep 15;370(2):198-207.

69 Dedinska I, Mikolajcik P, Skalova P, Mokan M, Laca L. Acute kidney injury after liver resection in elderly patients. BMC Nephrol. 2019 Jul 18;20(1):272.

70 Kaushal GP. Autophagy protects proximal tubular cells from injury and apoptosis. Kidney Int. 2012 Dec;82(12):1250-3.

71 Herzog C, Yang C, Holmes A, Kaushal GP. zVAD-fmk prevents cisplatin-induced cleavage of autophagy proteins but impairs autophagic flux and worsens renal function. Am J Physiol Renal Physiol. 2012 Oct 15;303(8): F1239-50.

72 Liu JX, Yang C, Zhang WH, Su HY, Liu ZJ, Pan Q, et al. Disturbance of mitochondrial dynamics and mitophagy in sepsis-induced acute kidney injury. Life Sci. 2019 Oct 15;235: 116828.

73 Li L, Wang ZV, Hill JA, Lin F. New autophagy reporter mice reveal dynamics of proximal tubular autophagy. J Am Soc Nephrol. 2014 Feb; 25(2):305-15.

74 Yang C, Wu HL, Li ZH, Chen XC, Su HY, Guo $\mathrm{XY}$, et al. Autophagy inhibition sensitizes renal tubular epithelial cell to $\mathrm{G} 1$ arrest induced by transforming growth factor beta (TGF-beta). Med Sci Monit. 2020 Jun 7;26:e922673.

75 Chen H, Fang Y, Wu J, Chen H, Zou Z, Zhang $\mathrm{X}$, et al. RIPK3-MLKL-mediated necroinflammation contributes to AKI progression to CKD. Cell Death Dis. 2018 Aug 29;9(9): 878.

76 Unno R, Kawabata T, Taguchi K, Sugino T, Hamamoto S, Ando R, et al. Deregulated MTOR (mechanistic target of rapamycin kinase) is responsible for autophagy defects exacerbating kidney stone development. Autophagy. 2020 Apr;16(4):709-23.

77 Tsuchie H, Miyakoshi N, Kasukawa Y, Nishi $\mathrm{T}$, Abe H, Segawa T, et al. The effect of teriparatide to alleviate pain and to prevent vertebral collapse after fresh osteoporotic vertebral fracture. J Bone Miner Metab. 2016 Jan;34(1): 86-91.

78 Denamur S, Tyteca D, Marchand-Brynaert J, Van Bambeke F, Tulkens PM, Courtoy PJ, et al. Role of oxidative stress in lysosomal membrane permeabilization and apoptosis induced by gentamicin, an aminoglycoside antibiotic. Free Radic Biol Med. 2011 Nov 1; 51(9):1656-65.
79 Denamur S, Boland L, Beyaert M, Verstraeten SL, Fillet M, Tulkens PM, et al. Subcellular mechanisms involved in apoptosis induced by aminoglycoside antibiotics: Insights on p53, proteasome and endoplasmic reticulum. Toxicol Appl Pharmacol. 2016 Oct 15;309: 24-36.

80 Pallet N, Bouvier N, Legendre C, Gilleron J, Codogno P, Beaune P, et al. Autophagy protects renal tubular cells against cyclosporine toxicity. Autophagy. 2008 Aug;4(6):783-91.

81 An N, Li ZH, Yang C, Pan QJ, Liu HF. Effect of cyclosporine A on autophagy-lysosomal pathway in tubular epithelial cells. Chinese Journal of Nephrology. 2017 Apr;33(4):2849

82 Yadav RK, Lee GH, Lee HY, Li B, Jung HE, Rashid HO, et al. TMBIM6 (transmembrane BAX inhibitor motif containing 6) enhances autophagy and reduces renal dysfunction in a cyclosporine A-induced nephrotoxicity model. Autophagy. 2015;11(10):1760-74.

83 Tang TT, Lv LL, Pan MM, Wen Y, Wang B, Li $\mathrm{ZL}$, et al. Hydroxychloroquine attenuates renal ischemia/reperfusion injury by inhibiting cathepsin mediated NLRP3 inflammasome activation. Cell Death Dis. 2018 Mar 2;9(3): 351.

84 Sperati CJ, Rosenberg AZ. Hydroxychloroquine-induced mimic of renal Fabry disease. Kidney Int. 2018 Sep;94(3):634.

85 Serre J, Buob D, Boffa JJ. Hydroxychloroquine-induced podocytopathy mimicking Fabry disease. BMJ Case Rep. 2019 May 13; 12(5):e228876.

86 Nath KA, Croatt AJ, Likely S, Behrens TW, Warden D. Renal oxidant injury and oxidant response induced by mercury. Kidney Int. 1996 Sep;50(3):1032-43.

87 Song XB, Liu G, Liu F, Yan ZG, Wang ZY, Liu $\mathrm{ZP}$, et al. Autophagy blockade and lysosomal membrane permeabilization contribute to lead-induced nephrotoxicity in primary rat proximal tubular cells. Cell Death Dis. 2017 Jun 8;8(6):e2863.

88 Lee WK, Probst S, Santoyo-Sánchez MP, AlHamdani W, Diebels I, von Sivers JK, et al. Initial autophagic protection switches to disruption of autophagic flux by lysosomal instability during cadmium stress accrual in renal NRK-52E cells. Arch Toxicol. 2017 Oct; 91(10):3225-45.

89 Skowyra ML, Schlesinger PH, Naismith TV, Hanson PI. Triggered recruitment of ESCRT machinery promotes endolysosomal repair. Science. 2018 Apr 6;360(6384):360.

90 Schmidt O, Teis D. The ESCRT machinery. Curr Biol. 2012 Feb 21;22(4):R116-20.

91 Radulovic M, Schink KO, Wenzel EM, Nähse $\mathrm{V}$, Bongiovanni A, Lafont F, et al. ESCRT-mediated lysosome repair precedes lysophagy and promotes cell survival. EMBO J. 2018 Nov 2;37(21):e99753.

92 McNally EK, Brett CL. The intralumenal fragment pathway mediates ESCRT-independent surface transporter down-regulation. Nat Commun. 2018 Dec 18;9(1):5358.
93 Fraiberg M, Elazar Z. A TRIM16-Galactin3 complex mediates autophagy of damaged endomembranes. Dev Cell. 2016 Oct 10 39(1):1-2.

94 Papadopoulos C, Kravic B, Meyer H. Repair or lysophagy: dealing with damaged lysosomes. J Mol Biol. 2020 Jan 3;432(1):231-9.

95 Mizushima N. The ubiquitin E2 enzyme UBE2QL1 mediates lysophagy. EMBO Rep. 2019 Oct 4;20(10):e49104.

96 Pogliani L. Molecular connectivity: treatment of the electronic structure of amino acids. J Pharm Sci. 1992 Oct;81(10):967-9.

97 Papadopoulos C, Kirchner P, Bug M, Grum D, Koerver L, Schulze N, et al. VCP/p97 cooperates with YOD1, UBXD1 and PLAA to drive clearance of ruptured lysosomes by autophagy. EMBO J. 2017 Jan 17;36(2):135-50.

98 Yoshida Y, Yasuda S, Fujita T, Hamasaki M, Murakami A, Kawawaki J, et al. Ubiquitination of exposed glycoproteins by SCF(FBXO27) directs damaged lysosomes for autophagy. Proc Natl Acad Sci USA. 2017 Aug 8;114(32):8574-9.

99 Maejima I, Takahashi A, Omori H, Kimura T, Takabatake Y, Saitoh T, et al. Autophagy sequesters damaged lysosomes to control lysosomal biogenesis and kidney injury. EMBO J. 2013 Aug 28;32(17):2336-47.

100 Steingrimsson E, Copeland NG, Jenkins NA. Melanocytes and the microphthalmia transcription factor network. Annu Rev Genet. 2004;38:365-411.

101 Inpanathan S, Botelho RJ. The lysosome signaling platform: adapting with the times. Front Cell Dev Biol. 2019;7:113.

102 Napolitano G, Ballabio A. TFEB at a glance. J Cell Sci. 2016 Jul 1;129(13):2475-81.

103 Zhao B, Dierichs L, Gu JN, Trajkovic-Arsic M, Axel Hilger R, Savvatakis K, et al. TFEBmediated lysosomal biogenesis and lysosomal drug sequestration confer resistance to MEK inhibition in pancreatic cancer. Cell Death Discov. 2020;6(1):12.

104 Sha Y, Rao L, Settembre C, Ballabio A, Eissa NT. STUB1 regulates TFEB-induced autophagy-lysosome pathway. EMBO J. 2017 Sep 1;36(17):2544-52.

105 Pan B, Zhang H, Cui T, Wang X. TFEB activation protects against cardiac proteotoxicity via increasing autophagic flux. J Mol Cell Cardiol. 2017 Dec;113:51-62.

106 Zhang W, Li X, Wang S, Chen Y, Liu H. Regulation of TFEB activity and its potential as a therapeutic target against kidney diseases. Cell Death Discov. 2020;6(1):32.

107 Zhao X, Chen Y, Tan X, Zhang L, Zhang H, $\mathrm{Li} Z$, et al. Advanced glycation end-products suppress autophagic flux in podocytes by activating mammalian target of rapamycin and inhibiting nuclear translocation of transcription factor EB. J Pathol. 2018 Jun; 245(2):235-48. 
108 Rega LR, Polishchuk E, Montefusco S, Napolitano G, Tozzi G, Zhang J, et al. Activation of the transcription factor EB rescues lysosomal abnormalities in cystinotic kidney cells. Kidney Int. 2016 Apr;89(4):862-73.

109 Zhu L, Yuan Y, Yuan L, Li L, Liu F, Liu J, et al. Activation of TFEB-mediated autophagy by trehalose attenuates mitochondrial dysfunction in cisplatin-induced acute kidney injury. Theranostics. 2020;10(13):5829-44.

110 Wang Y, Huang H, Jin Y, Shen K, Chen X, $\mathrm{Xu} Z$, et al. Role of TFEB in autophagic modulation of ischemia reperfusion injury in mice kidney and protection by urolithin A. Food Chem Toxicol. 2019 Sep;131:110591.

111 Zhang J, Wang J, Xu J, Lu Y, Jiang J, Wang $\mathrm{L}$, et al. Curcumin targets the TFEB-lyso- some pathway for induction of autophagy. Oncotarget. 2016 Nov 15;7(46):75659-71.

112 Moskot M, Montefusco S, Jakóbkiewicz-Banecka J, Mozolewski P, Węgrzyn A, Di Bernardo $\mathrm{D}$, et al. The phytoestrogen genistein modulates lysosomal metabolism and transcription factor EB (TFEB) activation. J Biol Chem. 2014 Jun 13;289(24):17054-69.

113 Brown RA, Voit A, Srikanth MP, Thayer JA, Kingsbury TJ, Jacobson MA, et al. mTOR hyperactivity mediates lysosomal dysfunction in Gaucher's disease iPSC-neuronal cells. Dis ModelMech.2019Oct 16;12(10):dmm038596.

114 Xu S, Li L, Li M, Zhang M, Ju M, Chen X, et al. Impact on autophagy and ultraviolet $B$ induced responses of treatment with the MTOR inhibitors rapamycin, everolimus,
Torin 1, and pp242 in human keratinocytes. Oxid Med Cell Longev. 2017;2017:5930639.

115 Zhang X, Chen W, Gao Q, Yang J, Yan X, Zhao $\mathrm{H}$, et al. Rapamycin directly activates lysosomal mucolipin TRP channels independent of mTOR. PLoS Biol. 2019 May; 17(5):e3000252.

116 Zhitomirsky B, Assaraf YG. Lysosomal sequestration of hydrophobic weak base chemotherapeutics triggers lysosomal biogenesis and lysosome-dependent cancer multidrug resistance. Oncotarget. 2015 Jan 20; 6(2):1143-56

117 Geisslinger F, Müller M, Vollmar AM, Bartel $\mathrm{K}$. Targeting lysosomes in cancer as promising strategy to overcome chemoresistance-A mini review. Front Oncol. 2020;10:1156. 